LA W RENCE LIVERM ORE NATIONAL LABORATORY

Computer Simulation of Blast Waves in a Tunnel with Sudden Decrease in Cross Section

L. A. Glenn, S. Neuscamman

August 29, 2011 
This document was prepared as an account of work sponsored by an agency of the United States government. Neither the United States government nor Lawrence Livermore National Security, LLC, nor any of their employees makes any warranty, expressed or implied, or assumes any legal liability or responsibility for the accuracy, completeness, or usefulness of any information, apparatus, product, or process disclosed, or represents that its use would not infringe privately owned rights. Reference herein to any specific commercial product, process, or service by trade name, trademark, manufacturer, or otherwise does not necessarily constitute or imply its endorsement, recommendation, or favoring by the United States government or Lawrence Livermore National Security, LLC. The views and opinions of authors expressed herein do not necessarily state or reflect those of the United States government or Lawrence Livermore National Security, LLC, and shall not be used for advertising or product endorsement purposes.

This work performed under the auspices of the U.S. Department of Energy by Lawrence Livermore National Laboratory under Contract DE-AC52-07NA27344. 


\title{
Computer Simulation of Blast Waves in a Tunnel with Sudden \\ Decrease in Cross Section
}

\author{
L. A. Glenn
}

S. Neuscamman

August 22, 2011

\begin{abstract}
The case of an explosion in a tunnel where the blast wave encounters a sudden decrease in cross section is studied with quasi-one-dimensional (1D) and two-dimensional axisymmetric codes (2D) and the results are compared to experimental data. It is found that the numerical results from both codes are in good agreement until the interface at the change in cross section is encountered. Thereafter, however, the peak pressure derived with the codes is found to be significantly higher than the experimental results although the agreement between the $2 \mathrm{D}$ result and the experiment improves with increasing distance down the tunnel. Peak pressure and impulse per unit area obtained downstream of the interface with the 1D analysis are found to be substantially higher than with either the experiment or the $2 \mathrm{D}$ results. The reason for this is the time delay for the shock reflecting off the (vertical) rigid wall between the inner and outer tunnel radii to interact with the (supersonic) core flow into the decreased cross section. In the 1D case the reflected and transmitted shocks are formed instantaneously across the entire cross section resulting in higher pressure and increased shock speed downstream of the interface.
\end{abstract}




\section{Introduction and Experimental Set-up}

This study was motivated by an experiment devised to study the effect of wall roughness on blast wave propagation in tunnels (Lunderman et al., 1993). A similar set of experiments was carried out in a smooth-wall system (Lunderman and Ohrt, 1997) and simulation of these experiments with quasi-1D and 2D axisymmetric codes produced excellent agreement with the observed results (Glenn et al., 2011; Neuscamman et al., 2011). In these latter experiments, a small spherical charge was detonated in a smooth pipe of uniform cross section and pressure history measurements were made along the wall at various distances from the shot point. In the former case a slightly different setup was employed as illustrated in figure 1.

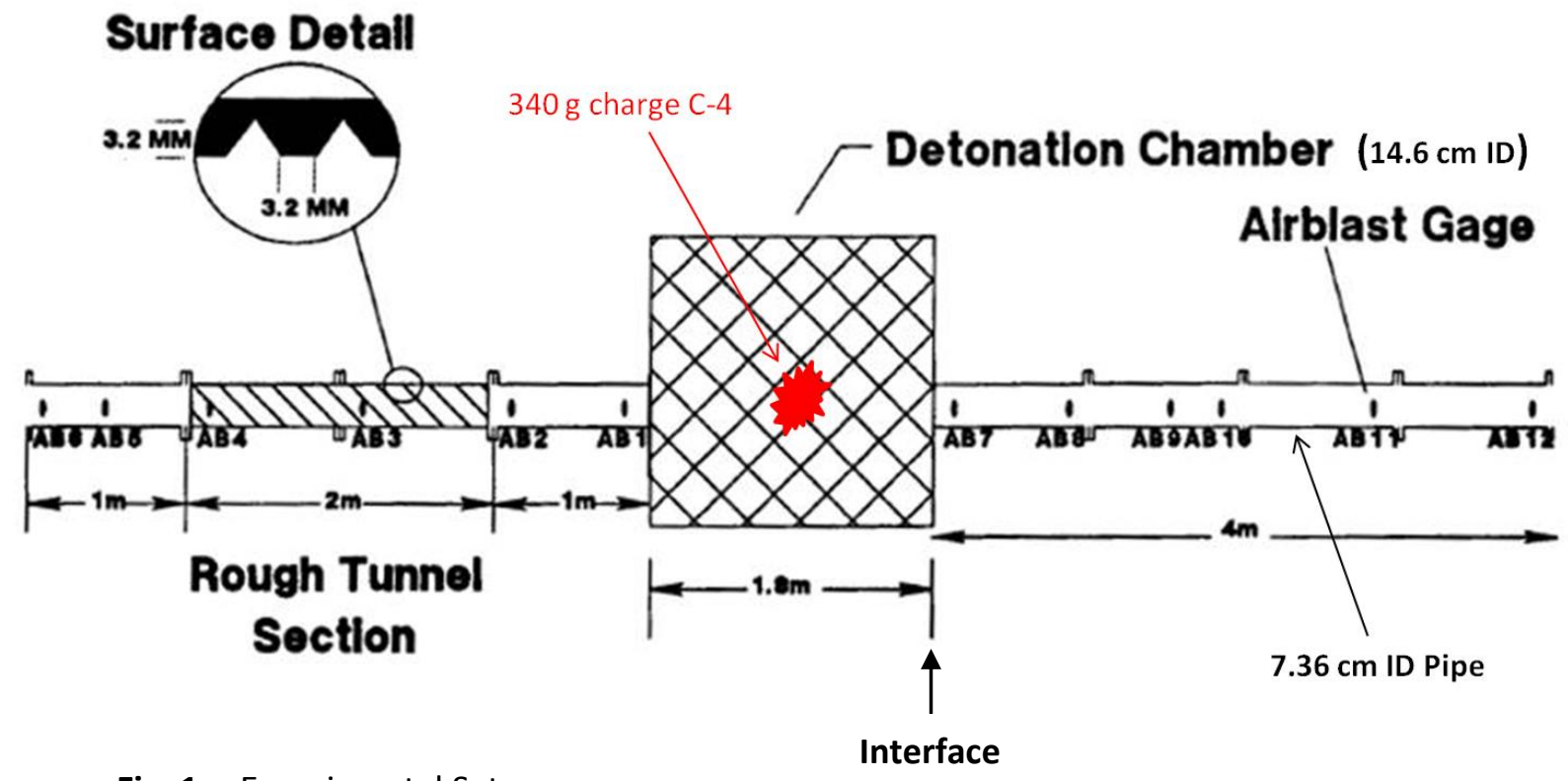

Fig. 1 Experimental Setup

In this case a $340 \mathrm{~g}$ charge of C4 explosive, $5.04 \mathrm{~cm} \mathrm{X} 5.04 \mathrm{~cm} \mathrm{X} 8.23 \mathrm{~cm}$, was centered in a $1.8 \mathrm{~m}$ long section of heavy duty steel pipe with an internal diameter of $14.6 \mathrm{~cm}$ (the detonation chamber). Connected to one side were 41 -m long segments of smooth Schedule 80 steel pipe with an internal diameter of $7.36 \mathrm{~cm}$. On the other side a similar arrangement was used except that the center 2 segments were replaced with Schedule 160 (6.67 cm inner diameter) pipe which allowed deep grooves to be machined into the (thicker) walls. For the purposes of this study, the focus will be on the righthand-section of figure 1 and the fourfold decrease in cross section that occurs at the end of the detonation chamber. As shown in the figure, airblast gages were located at $109 \mathrm{~cm}(A B 7), 171 \mathrm{~cm}(A B 8)$, $271 \mathrm{~cm}(A B 9), 371 \mathrm{~cm}(A B 10)$, and $471 \mathrm{~cm}(A B 12)$; the gage $A B 11$ at $440 \mathrm{~cm}$ failed for this test. 


\section{Codes Employed for the Analysis}

For the quasi-1D calculations the Sphere and TUNnel (STUN) code was employed. This code was developed initially to model nuclear explosions in tunnels (Glenn, 2001) and was based on earlier work that simulated the performance of an impulsive water cannon (Glenn, 1975), blast wave attenuation using water jets (Glenn, 1980; 1982) and the modeling of hypervelocity launchers (Glenn et al., 1989; Glenn, 1990).

The 2D axisymmetric calculations were carried out with the ALE3D code (Nichols ed., 2009), developed at the Lawrence Livermore National Laboratory, and widely used by both the DOD and DOE communities. ALE3D is a three-dimensional code that uses arbitrary Lagrange-Eulerian grid motion to efficiently perform continuum mechanics simulations on massively parallel computing platforms. It should be noted in passing that fully 3D simulations of the problem under discussion in this report were also carried out and the results were consistent with the 2D axisymmetric calculations.

\section{Simulation Results}

Figures 1 and 2 show the computed pressure and impulse per unit area computed at 4 equally spaced positions in the explosion chamber (no gages were located at these positions so no comparison can be made with experiment). The left hand panels compare the pressure history derived from the STUN calculations with the pressure data from ALE3D averaged over the lateral cross section at each position. The right hand panels show the impulse and both the average ALE3D results and the centerline (axis) impulse are presented (the latter with the hairline red curve) to give an indication of the lateral variation in the 2D calculation; the centerline pressure data are suppressed in the left hand panels for clarity.

It is observed that there is good agreement between the STUN and ALE3D results and the agreement actually improves as the shock travels towards the interface with the test section.

Figures 3 through 5 are in similar format however these show the predicted results in the tunnel test section and include the experimental data. At the first location, $19 \mathrm{~cm}$ beyond the interface $($ at $109 \mathrm{~cm})$, the STUN and ALE3D results are still in fairly good agreement but both overestimate the pressure peaks seen in the experiment and the times of arrival of the later peaks are also inconsistent with observation. At the next location, $171 \mathrm{~cm}$, the pressure peaks predicted by STUN are consistently higher than the ALE3D results and although the latter are higher than the data indicate, the integrated impulse per unit area calculated with ALE3D agrees fairly well with experiment. Further downstream the discrepancy between the STUN results and the other 2 only increases.

\section{Analysis}

The reason for the discrepancy between the STUN and ALE3D results after the blast wave enters the tunnel test section is illustrated in figure 6 , which shows a sequence of synthetic Schlieren images derived by plotting the gradient of pressure just prior to, during, and after the shock hits the interface. 

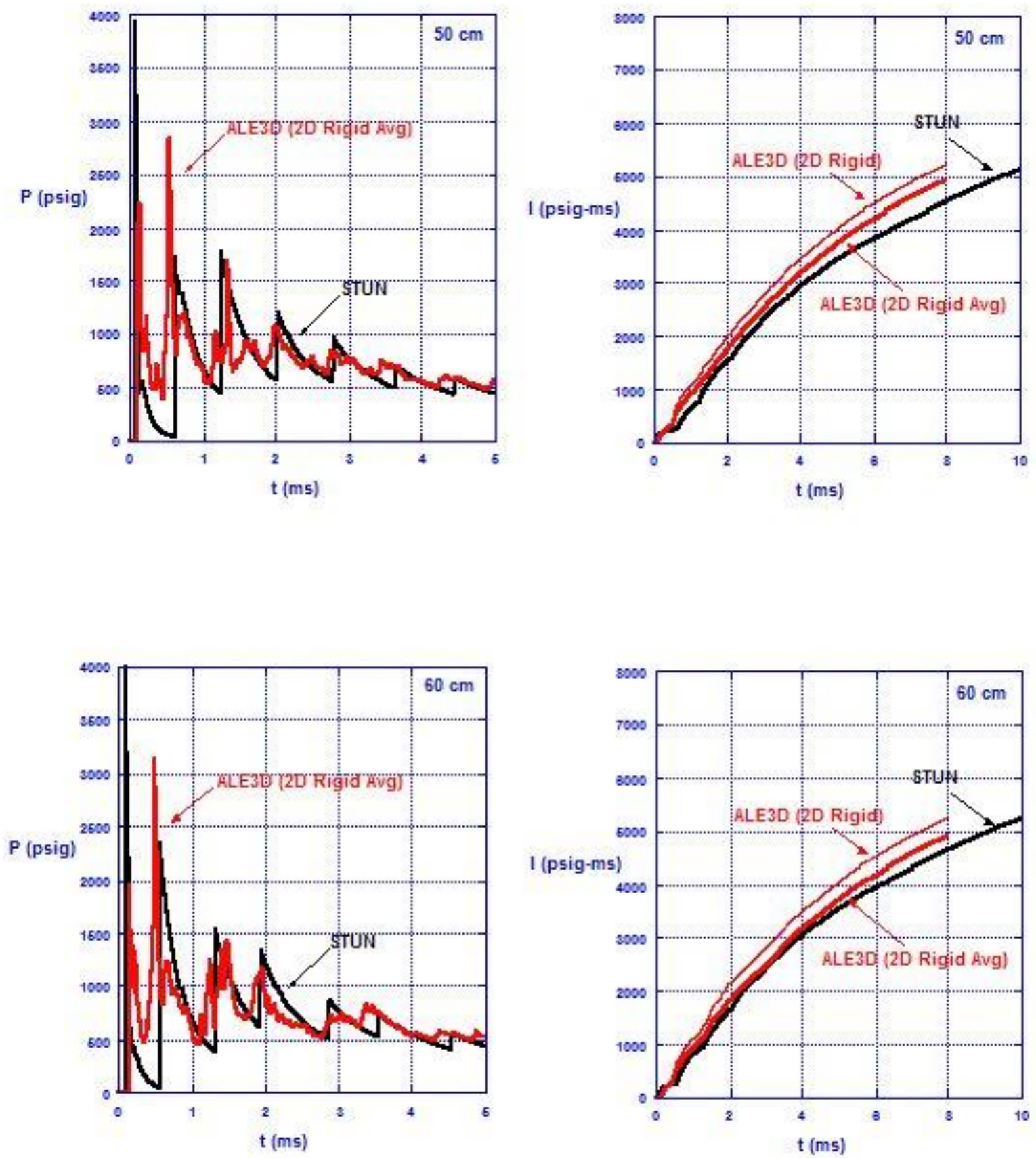

Fig. 1 Pressure and impulse per unit area as a function of time at positions 50 and $60 \mathrm{~cm}$ from the plane of the explosion (in the detonation chamber). 

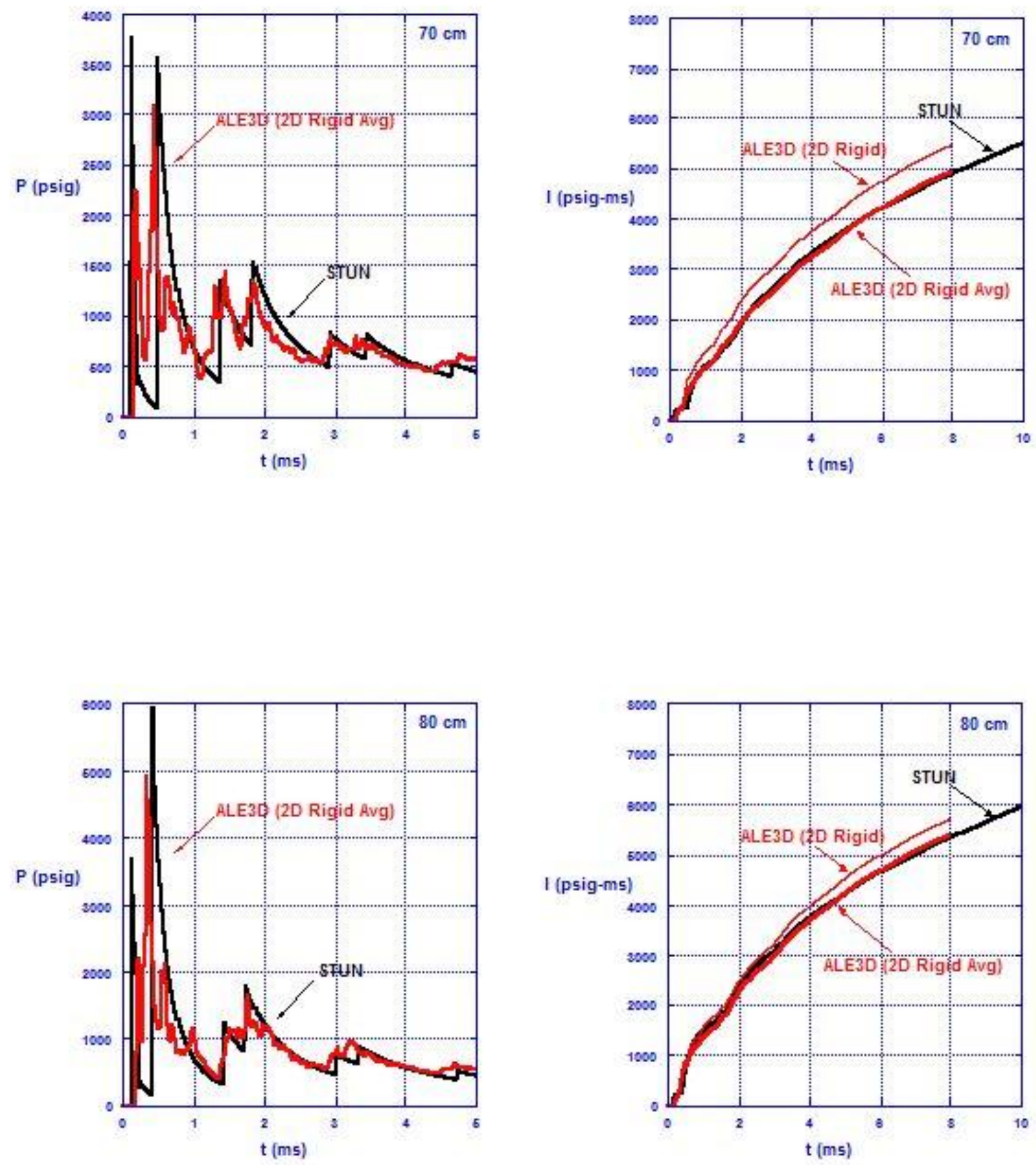

Fig. 2 Pressure and impulse per unit area as a function of time at positions 70 and $80 \mathrm{~cm}$ from the plane of the explosion (in the detonation chamber). 

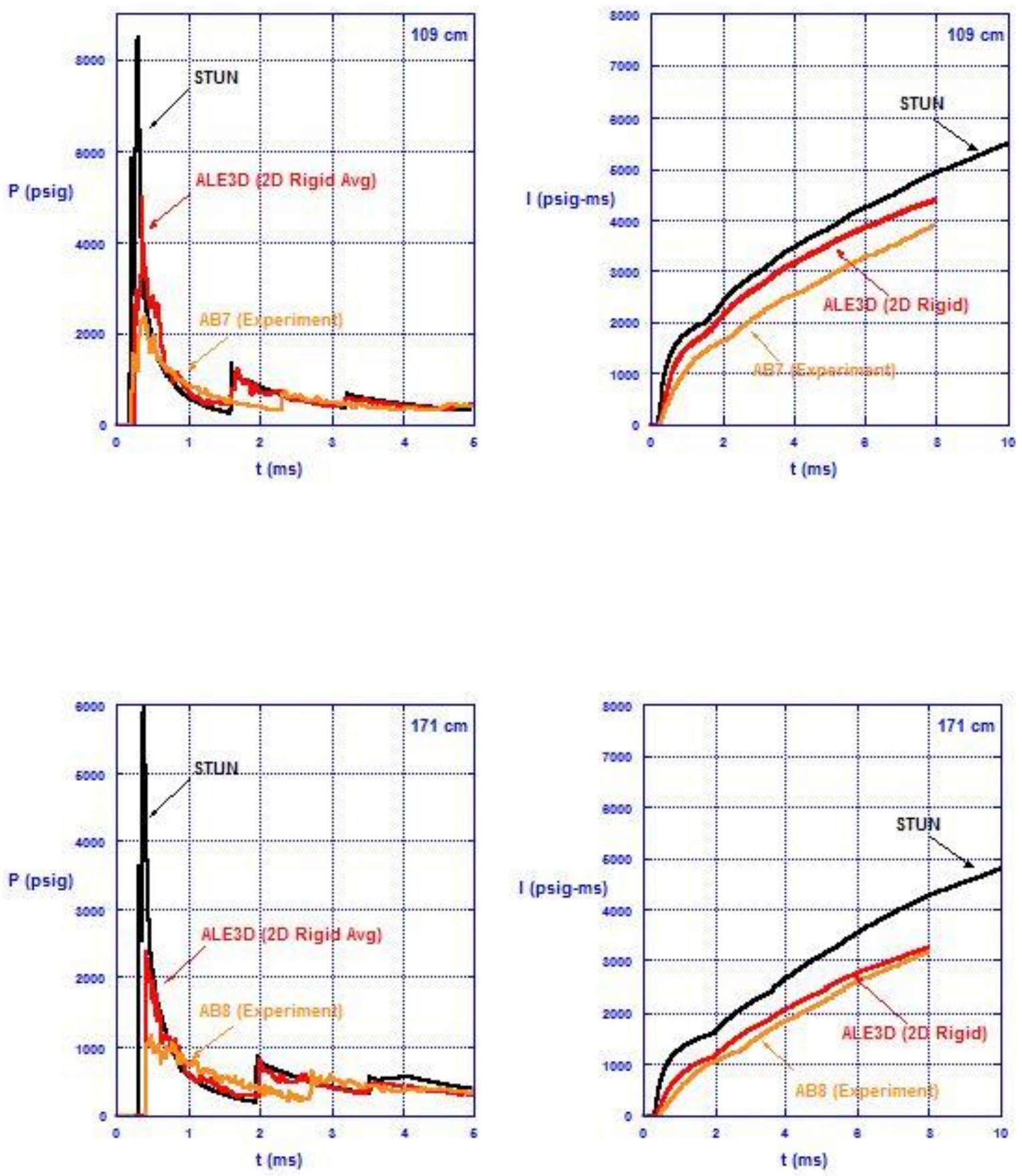

Fig. 3 Pressure and impulse per unit area as a function of time at positions 109 and $171 \mathrm{~cm}$ from the plane of the explosion (in the tunnel test section). 

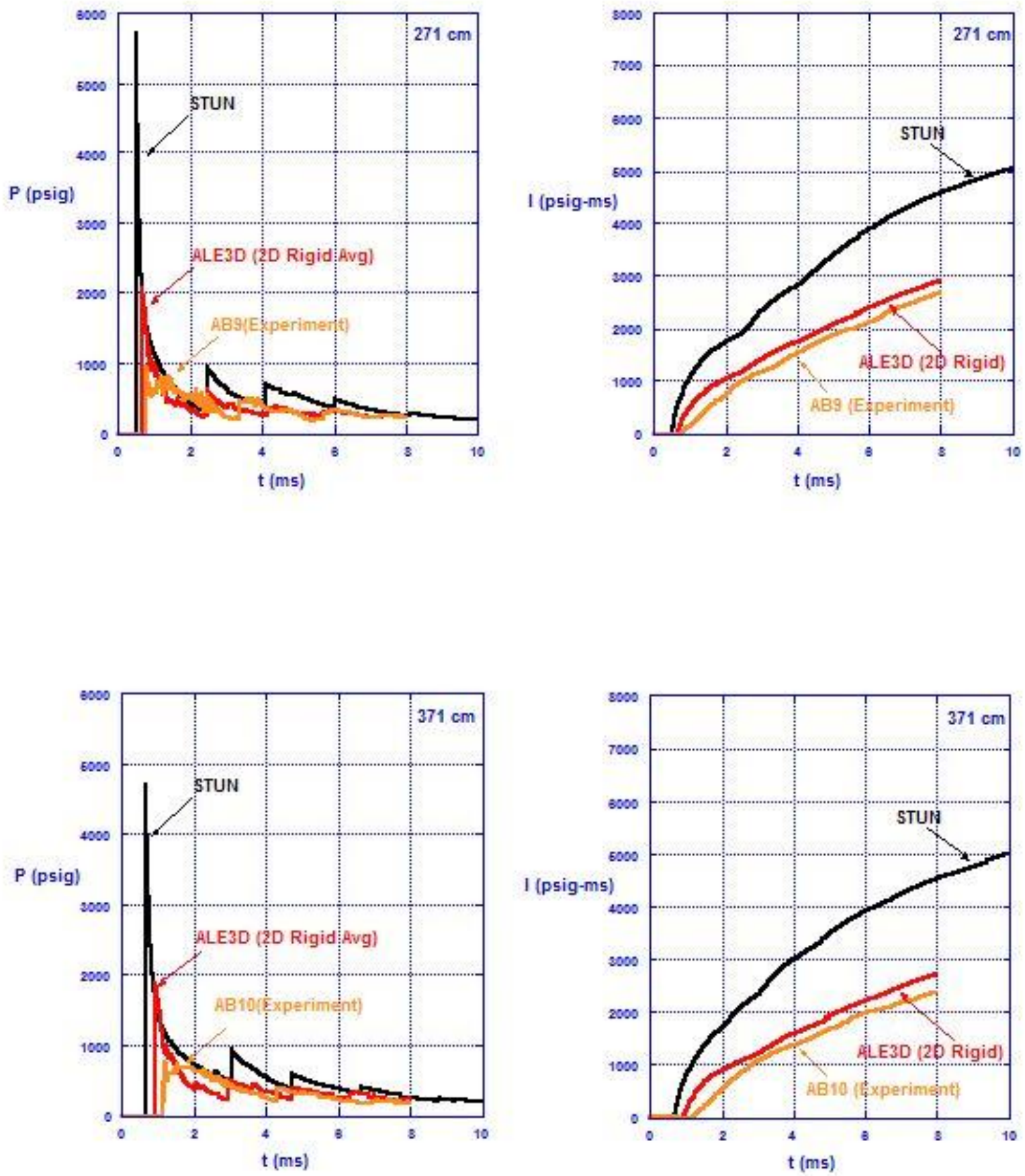

Fig. 4 Pressure and impulse per unit area as a function of time at positions 271 and $371 \mathrm{~cm}$ from the plane of the explosion (in the tunnel test section). 

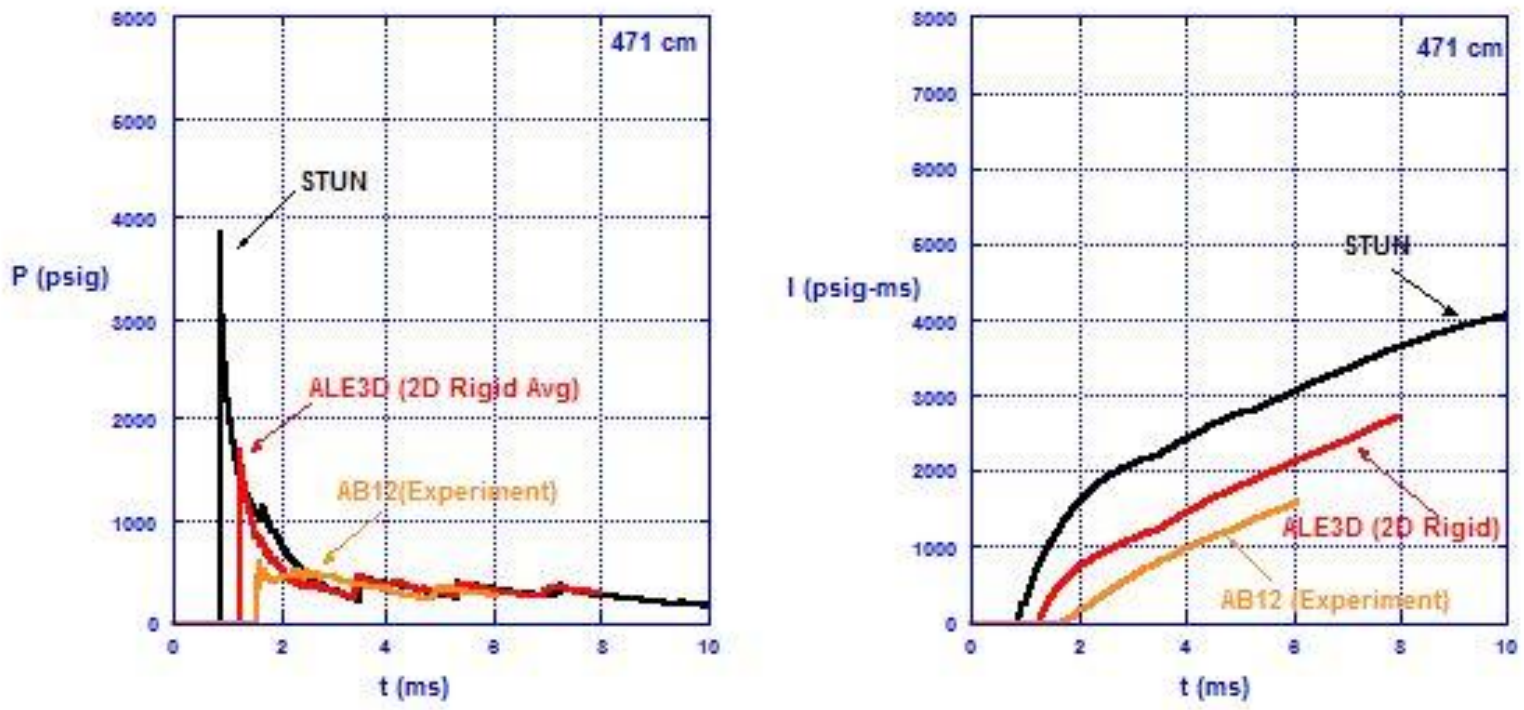

Fig. 5 Pressure and impulse per unit area as a function of time at position $471 \mathrm{~cm}$ from the plane of the explosion (in the tunnel test section).

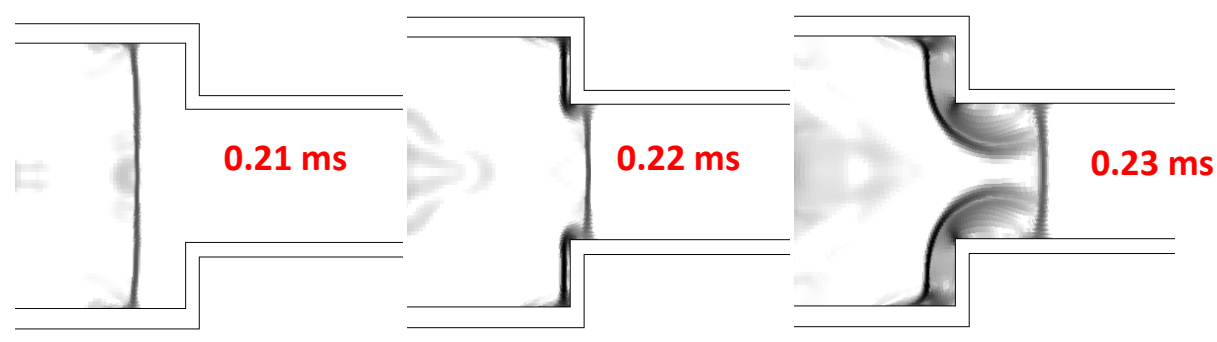

Fig. 6 Synthetic Schlieren images of ALE3D simulation just prior to, during, and after blast wave enters tunnel test section. 
At $0.21 \mathrm{~ms}$ a nearly normal shock is approaching the tunnel test section. $0.01 \mathrm{~ms}$ later an even stronger shock is seen to reflect off the vertical end plate of the detonation chamber. And still $0.01 \mathrm{~ms}$ later this reflected shock moves out more or less spherically from the corner of the interface and begins to interact with the core flow in the tunnel test section. However the axial flow behind the initial shock is supersonic so that the leading segment of core flow in the tunnel is unaffected by the reflected shock; as seen in the last panel of figure 6 it takes finite time for the latter to completely interact with the core section. Figure 7 show the local axial Mach number as a function of distance, as computed by STUN, prior to interaction with the interface $($ at $90 \mathrm{~cm}$ ) and subsequent to this interaction (at times of 0.15 and $0.25 \mathrm{~ms})$.

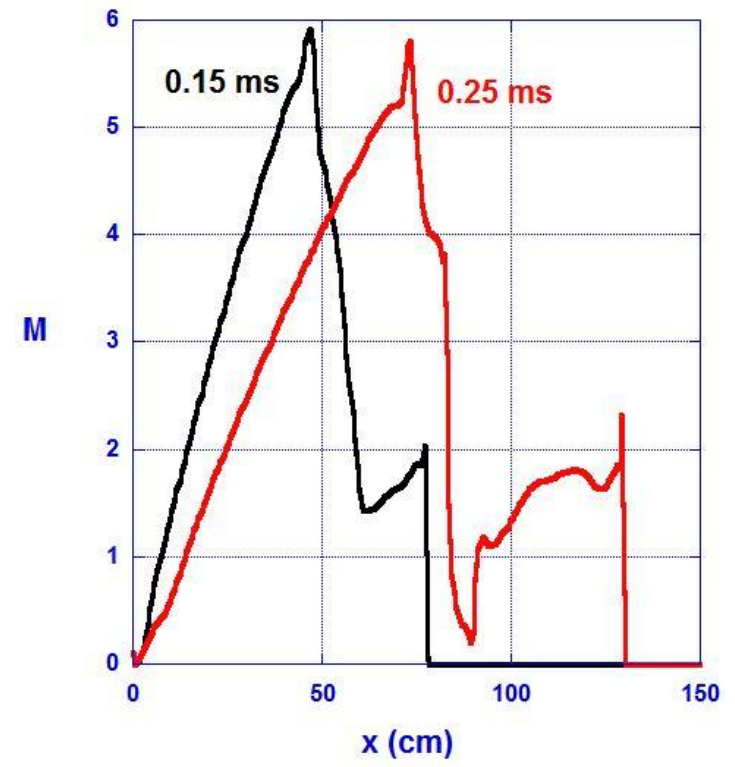

Fig. 7 Local Mach number as a function of distance from the explosion, derived from the STUN simulation.

It can be seen that $M$ varies from 2 to 6 . And in STUN the key point is that when the shock hits the interface the effect on the core flow is instantaneous as there can be no lateral variation in the 1D uniaxial flow. Since the reflected shock pressure is much higher than behind the incident shock the leading segment of the core flow is accelerated more than in the 2D case (and in the experiment) so the arrival time of the shock down the tunnel test section will be shorter and the pressure recorded will be greater. Moreover, the reflected shock in the detonation chamber will be stronger as it moves back towards the plane of detonation and reflects there from. The subsequent periodic spikes in pressure seen prominently in figures 4 and 5 are a direct result of these reflections and are responsible for the increasing discrepancy between the impulse calculated with STUN and ALE3D. The discrepancy begins to diminish at the last gage position (figure 5 ) because this is close to the open end of the tunnel test section and the rarefactions that reflect from that position effectively reduce the pressure spikes.

It should be noted that there is another position within the setup as displayed in figure 1 in which there is a sudden decrease in cross section, namely at the intersection of the symmetry plane passing through 
the explosive charge and the wall of the detonation chamber. The flow cross section around the charge is spherical (hemispherical in the symmetry context) and varies as $r^{2}$, reaching a maximum value of $2 \pi R^{2}$ at the wall of the chamber $(R=7.3 \mathrm{~cm})$. Immediately thereafter the flow is axial and the cross section is $\pi R^{2}$ until the intersection of the detonation chamber with the tunnel test section. In this case, however, the factor of 2 sudden decrease in cross section is mitigated by the fact that the velocity vectors around the charge are not directed uniaxially. Although there is some initial motion directly down the axis of the detonation chamber, most of the motion is initially directed at the walls and there is considerable lateral reflection, quite distinct from the images in figure 6 where a uniaxially directed shock approaches and interacts with change in cross section. Figures 1 and 2 show that by the time the shock reaches the $50 \mathrm{~cm}$ position $(x / R=6.85)$ agreement between the STUN and ALE3D impulse calculations is already good and at the $80 \mathrm{~cm}$ position $(x / R=11.0)$ the 2 calculations are virtually indistinguishable.

The most likely explanation for the discrepancy between the experimental results and the simulations (arrival times and diminished peaks) is the gamma law equation of state both codes used for the air. At $0.15 \mathrm{~ms}$, the STUN calculation showed the temperature at the shock front to be well in excess of $10^{4} \mathrm{~K}$. Under these conditions, the air would be fully dissociated and partially ionized so the effective ratio of specific heats would likely differ significantly from ideal gas behavior.

\section{Conclusion}

The case of an explosion in a tunnel where the blast wave encounters a sudden decrease in cross section was studied with quasi-one-dimensional (1D) and two-dimensional axisymmetric codes (2D) and the results were compared to experimental data. It was found that the numerical results from both codes were in good agreement until the interface at the change in cross section was encountered. Thereafter, however, the peak pressure derived with the codes was found to be significantly higher than the experimental results although the agreement between the 2D result and the experiment improved with increasing distance down the tunnel. Peak pressure and impulse per unit area obtained downstream of the interface with the $1 \mathrm{D}$ analysis were found to be substantially higher than with either the experiment or the $2 \mathrm{D}$ results. The reason for this is the time delay for the shock reflecting off the (vertical) rigid wall between the inner and outer tunnel radii to interact with the (supersonic) core flow into the decreased cross section. In the 1D case the reflected and transmitted shocks are formed instantaneously across the entire cross section resulting in higher pressure and increased shock speed downstream of the interface.

Although the 1D results overestimate the downstream effects of the blast on the configuration studied, the STUN code has typically been employed to study blast waves in tunnels with no change in cross section (for which the results have been shown to be remarkably accurate) or when the downstream cross section is reduced by a tunnel blockage such as a train. In this case, the flow between the train and tunnel walls is in an annulus for which the time delay cited above should be much less. It is easy to show, for example, that with a downstream to upstream fractional annular cross section equal to 0.25 , the ratio of time delays in the annular case to that in the circular case is $2-\sqrt{3}=0.27$. Moreover, the annular gap is often smaller than this. On the other hand, the ratio of surface areas is $2+\sqrt{3}=3.73$ so 
that frictional drag on the annular walls will be increased which will tend to increase the pressure in the annulus; this latter effect is incorporated in the STUN calculation but cannot be easily accounted for in ALE3D.

Also, the 1D calculation in this study was carried out on a PC in less than a minute of clock time whereas the $2 \mathrm{D}$ calculation required over 18 hours ( 6 hours of clock time with 3 processors). Finally, it should be noted that the problem encountered here with step change in cross section does not occur when the change in cross section is more gradual as in a convergent nozzle and the 1D approach has been shown to quite accurately predict compressible flows in that geometry.

\section{References}

Glenn, L. A., STUN Simulation of Lunderman \& Ohrt Experiment, Lawrence Livermore National Laboratory Presentation LLNL-PRES-479948, 6 April 2011.

Glenn, L. A., Simulating MARVEL with the STUN Code, Technical Report UCRL-ID-143993, Lawrence Livermore National Laboratory, 6 June 2001.

Glenn, L. A., Design Limitations on Ultra-High Velocity Projectile Launchers, Int. J. Impact Engrg., 10, 185196, 1990.

Glenn, L. A., Latter, A. L., and E. A. Martinelli, Multistage Gasdynamic Launchers, Shock Waves in Condensed Matter - 1989, Proceedings of the Topical Conference on Shock Waves in Condensed Matter, Albuquerque, NM, 14-17 August; see also Lawrence Livermore National Laboratory Report UCRL100586, 1989.

Glenn, L. A., Blast Attenuation in a Shot Tunnel using Water Jets, Technical Report UCRL-19419, Lawrence Livermore National Laboratory, 2 July 1982.

Glenn, L. A., Divergent Impulsive Crossflow over Packed Columnar Arrays, Nucl. Engrg. And Des., 56, 429437, 1980.

Glenn, L. A., The Mechanics of the Impulsive Water Cannon, Computers and Fluids, 3, 197-215, 1975.

Lunderman, C. V., Welch, C. R., Joachim, C. E. and J. S. Furey, Effects of Tunnel Wall Roughness on Airblast from Internal Detonations-Results from Small-Scale Experiments, $64^{\text {th }}$ Shock and Vibration Symposium, Fort Walton Beach, FL, 25-28 October 1993.

Lunderman, C. V. and A. P. Ohrt, Small-Scale Experiments of In-Tunnel Airblasts from Internal and External Detonations, $8^{\text {th }}$ International Symposium on Interactions of the Effects of Munitions with Structures, McLean, VA, 21-25 April 1997. 
Neuscamman, S, Glenn, L. A., Schebler, S., McMichael, L., and L. Glascoe, Simulating Small-Scale Experiments of In-Tunnel Airblast using STUN and ALE3D, Internal Technical Report, Lawrence Livermore National Laboratory, 22 June 2011.

Nichols, A. L. III (ed.), Users Manual for ALE3D, An Arbitrary Lagrange/Eulerian 2D and 3D Code System, Lawrence Livermore National Laboratory Report UCRL-SM- 404490 Rev 1, June 2009. 